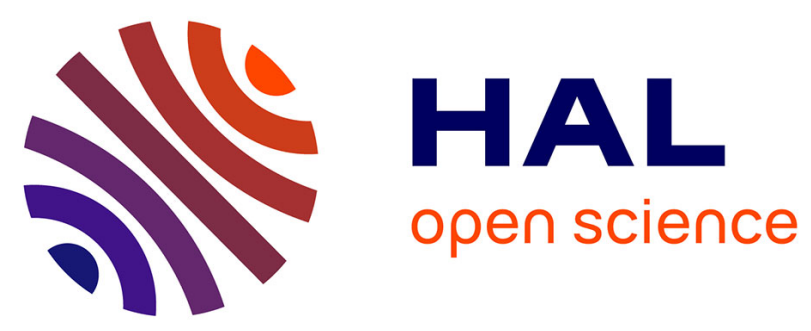

\title{
Fast Modular Arithmetic on the Kalray MPPA-256 Processor for an Energy-Efficient Implementation of ECM
}

Masahiro Ishii, Jérémie Detrey, Pierrick Gaudry, Atsuo Inomata, Kazutoshi Fujikawa

\section{To cite this version:}

Masahiro Ishii, Jérémie Detrey, Pierrick Gaudry, Atsuo Inomata, Kazutoshi Fujikawa. Fast Modular Arithmetic on the Kalray MPPA-256 Processor for an Energy-Efficient Implementation of ECM. IEEE Transactions on Computers, 2017, 66 (12), pp.2019-2030. 10.1109/TC.2017.2704082 . hal01299697v2

\section{HAL Id: hal-01299697 \\ https://hal.inria.fr/hal-01299697v2}

Submitted on 25 Jul 2017

HAL is a multi-disciplinary open access archive for the deposit and dissemination of scientific research documents, whether they are published or not. The documents may come from teaching and research institutions in France or abroad, or from public or private research centers.
L'archive ouverte pluridisciplinaire HAL, est destinée au dépôt et à la diffusion de documents scientifiques de niveau recherche, publiés ou non, émanant des établissements d'enseignement et de recherche français ou étrangers, des laboratoires publics ou privés. 


\title{
Fast Modular Arithmetic on the Kalray MPPA-256 Processor for an Energy-Efficient Implementation of ECM
}

\author{
Masahiro Ishii, Jérémie Detrey, Pierrick Gaudry, Atsuo Inomata, and Kazutoshi Fujikawa
}

\begin{abstract}
The Kalray MPPA-256 processor is based on a recent low-energy manycore architecture. In this article, we investigate its performance in multiprecision arithmetic for number-theoretic applications. We have developed a library for modular arithmetic that takes full advantage of the particularities of this architecture. This is in turn used in an implementation of the ECM, an algorithm for integer factorization using elliptic curves. For parameters corresponding to a cryptanalytic context, our implementation compares well to state-of-the-art implementations on GPU, while using much less energy.
\end{abstract}

Index Terms-Kalray MPPA-256 manycore processor, Multiprecision modular arithmetic, Integer factorization, Elliptic curve method.

\section{INTRODUCTION}

I NVENTED in 1985 by Lenstra [1], the elliptic curve method (ECM) is an integer factoring algorithm that is today considered the best one when one wants to extract prime factors of moderate size in a large number. It is therefore the method of choice when one wants to check if a number is smooth (i.e., if all its prime factors are below a certain bound). It is also used as one of the steps in the factorization toolchain in general-purpose computer algebra systems such as Sage, GP/Pari, Magma or Maple. The widespread GMP-ECM [2] is a reference implementation in this context; more recent libraries like EECM-MPFQ [3] make use of the faster elliptic curve arithmetic provided by the so-called twisted Edwards curves, instead of the traditional Montgomery model.

As a smoothness test, ECM is also an important subroutine for more general algorithms. We focus here on ECM parameters that are relevant in the context of the number field sieve (NFS) for integer factorization or for computing discrete logarithms in large-characteristic finite fields [4]. In NFS, a large proportion of the time is spent looking for relations, which can be done by sieving or by ECM, and more generally with a combination of these two strategies. In NFS variants that yield the best asymptotical complexities, namely Coppersmith's multiple polynomial NFS [5], or batch NFS [6], the role of ECM in the relation collection step is even more important. For a 768-bit integer handled with NFS, ECM is run on inputs that have typically around 200 bits, and the smoothness bound has about 35 bits.

Apart from the relation collection step, ECM is also important in the final step of NFS for discrete logarithms,

- M. Ishii is with the Tokyo Institute of Technology, Tokyo, Japan. E-mail: mishii@c.titech.ac.jp

- J. Detrey and P. Gaudry are with LORIA (INRIA, CNRS and Université de Lorraine), Nancy, France.

E-mail: $\{$ jeremie.detrey, pierrick. gaudry\}@ioria.fr

- A. Inomata and K. Fujikawa are with the Information Initiative Center, Nara Institute of Science and Technology, Nara, Japan.

E-mail: $\{$ atsuo, fujikawa\}@itc.naist.jp

Manuscript received XXXX; revised XXXX. called the individual logarithm step, where a descent phase is initialized using a smoothness test. Here, the input can have up to 500 bits, and the smoothness bound is also larger, but there is still not enough published data on the topic to be precise. In a LogJam-type attack [7], assuming the large precomputation has been done, this smoothing step with ECM is the bottleneck.

In those two contexts related to NFS, the quantity of numbers to be tested for smoothness is huge, but this is a task that can be parallelized in a straightforward way. This is the reason why a lot of effort has been put in decreasing the cost of ECM for numbers of moderate sizes, in particular using non-general-purpose coprocessors. In [8], Bos and Kleinjung optimized ECM using twisted Edwards curves on GPU. This was further improved in [9] and provides the most efficient implementation so far for the NFS context, using algorithmic improvements to fit the memory constraints of a GPU environment.

In this paper, we explore the potential of the MPPA-256 processor developed by Kalray [10] as an ECM coprocessor. This chip, whose name stands for Massively Parallel Processor Array, is a recently designed, lightweight manycore processor, where each of the 256 cores is an independent 32-bit VLIW (Very Long Instruction Word) architecture. In the ECM algorithm, most of the time is spent in the elliptic curve group law, that must be performed modulo the integer that is being factored. Therefore, in the end, most of the time is spent doing multiprecision modular arithmetic, in particular modular multiplications, and this operation must be optimized as much as possible.

We propose a library for multiprecision arithmetic for numbers of fixed sizes corresponding to our target in the NFS context, where all critical parts are written in assembly, taking full advantage of the VLIW architecture to explicitly schedule the operations in all available pipelines. On top of it we implemented the ECM algorithm, following the algorithmic ideas of [9], that we slightly improved. The memory constraints of a GPU and of the MPPA-256 are 
rather different, but the same strategies behave pretty well.

The results are quite satisfactory: in terms of number of curves tried per second on the whole chip, the GPU is faster than the MPPA-256 by a factor around 3, but this must be put in a larger perspective since the peak power consumption of the MPPA-256 is only $16 \mathrm{~W}$, while the GPU needs a bit less than $250 \mathrm{~W}$. So, in terms of number of curves tried per joule, the count is in favor of the MPPA-256 by a factor ranging from 5 to 7 , depending on the context.

The source code written for all our experiments is distributed under a free-software license and can be downloaded from https://gforge.inria.fr/projects/kalray-ecm. Although the ECM part is admittedly quite specialized, the multiprecision modular arithmetic library can be used in other contexts.

The paper is organized as follows. In the next section, we start with a description of the MPPA-256 processor, where we insist in particular on the architecture of the individual cores. Then, in Section 3, we explain our low-level implementation of the multiprecision modular arithmetic library. Finally, Section 4 contains details about the ECM applications, with benchmarks and a comparison with the literature.

\section{The Kalray MPPA-256 manycore Proces- SOR}

\subsection{Global overview}

Launched in 2012, the Kalray MPPA-256 processor (codenamed Andey) is a single $28 \mathrm{~nm}$ CMOS chip, clocked at $400 \mathrm{MHz}$, which integrates a $4 \times 4$ array of 16 -core compute clusters (CCs), along with 4 quad-core I/O subsystems located on the north, south, east and west ends of the chip, all connected by means of two toric networks-on-chip (NoCs), as depicted in Figure 1. The I/O subsystems allow one to interface the MPPA-256 chip with a host CPU, using PCI Express or Ethernet connections, for instance.

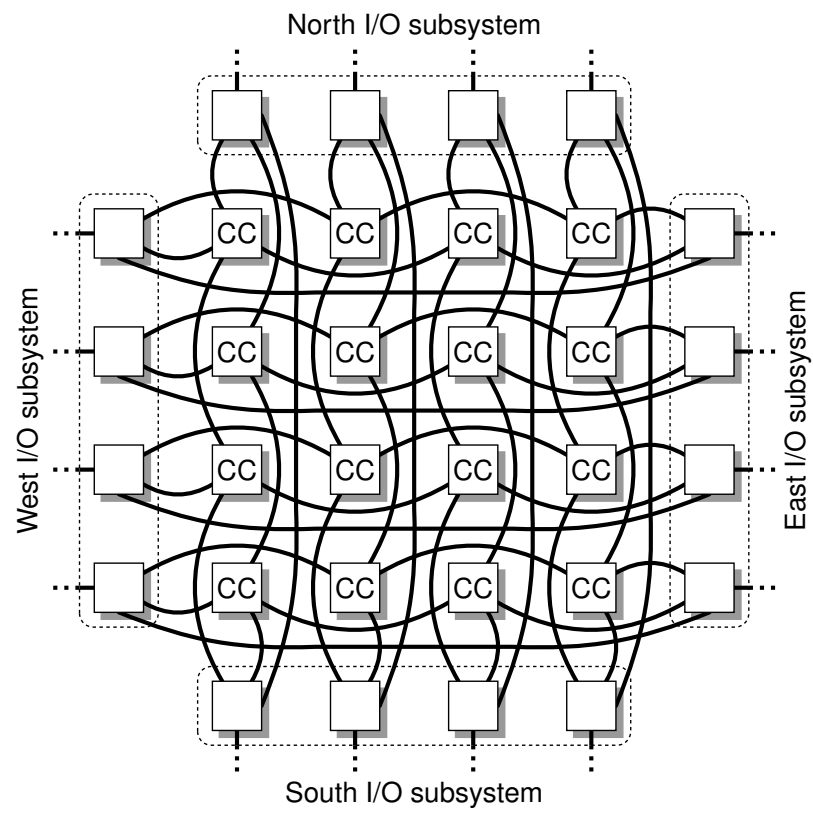

Fig. 1. Global architecture of the Kalray MPPA-256 [11].
Each compute cluster is composed of 16 cores, or processing engines (PEs), along with an extra core, the resource manager (RM), reserved for system use, and a 2 MB memory bank, shared by the 17 cores. A schematic view of a compute cluster is given in Figure 2.

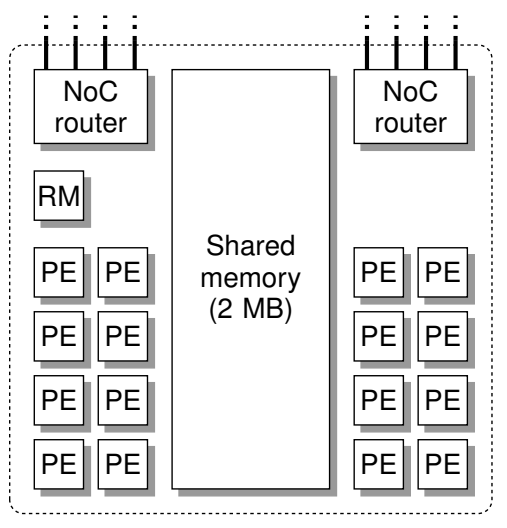

Fig. 2. Details of a compute cluster [11].

Each core of the I/O subsystems runs under the RTEMS ${ }^{1}$ real-time operating system, while the RM of each compute cluster runs under NodeOS, a specific operating system developed by Kalray. Both RTEMS and NodeOS implement POSIX-compatible APIs. MPPA-256 applications are then designed as POSIX-like processes deployed on the I/O subsystems and on the compute clusters, communicating together through the NoCs using network operations similar to reads and writes on UNIX sockets. Finally, a Pthreads-like interface allows one to run up to 16 threads in parallel on each compute cluster, thanks to their multi-core architecture.

\subsection{Core architecture}

The cores in the MPPA-256 are all based on the Kalray-1 (or K1) microarchitecture. It is an in-order, fully-pipelined, 32-bit, VLIW (Very Long Instruction Word) processor, which embeds five execution units: two Arithmetic \& Logic Units $\left(\mathrm{ALU}_{0}\right.$ and $\left.\mathrm{ALU}_{1}\right)$, a Multiply-Accumulate Unit (MAU), a Load/Store Unit (LSU), and a Branch \& Control Unit (BCU). The MAU can as well serve as Floating-Point Unit (FPU), and both the MAU and the LSU also support a subset of the ALU instruction set (referred to as $A L U_{\text {tiny }}$ ).

These execution units communicate by means of a shared register file (RF) of 64 32-bit general-purpose registers, which supports up to 11 read and 4 write accesses per cycle. In case of read-after-write dependencies, the register file can be bypassed, and the output of one unit directly used as the input of another one, so as to save one clock cycle between consecutive dependent instructions.

Finally, each $\mathrm{K} 1$ core has dedicated instruction and data caches of $8 \mathrm{kB}$ each, along with a 64 -byte write buffer.

The microarchitecture, along with a schematic representation of the pipeline stages, are depicted in Figure 3.

1. Real-Time Executive for Multiprocessor Systems, https:/ /www.rtems. org/. 


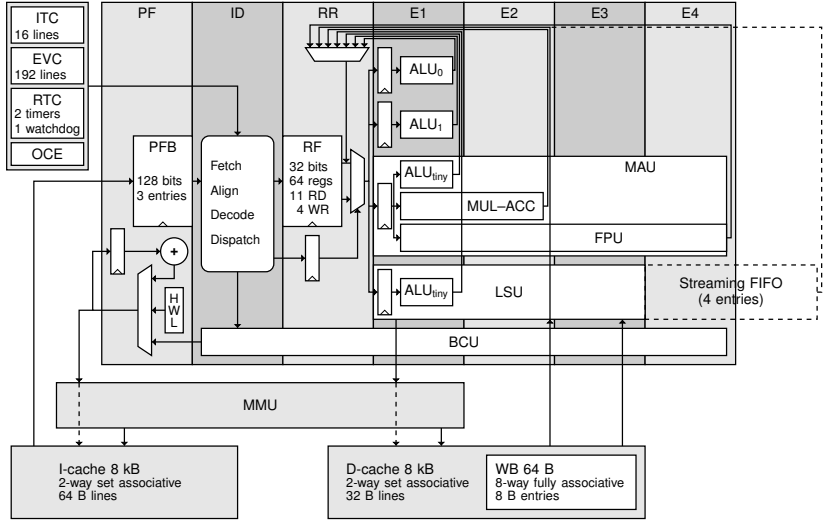

Fig. 3. VLIW pipeline of the K1 architecture [12].

\subsection{The Kalray-1 instruction set}

The $A L U_{\text {tiny }}$ instruction set, which is supported by both ALUs, along with the MAU and the LSU, covers most of the simple 32-bit integer operations, such as addition, subtraction and bitwise logic. The main ALUs also support a few extra integer instructions (such as shifts), and can even be combined to support 64-bit instructions, operating on pairs of registers. All these ALU instructions have a 1-cycle latency.

The MAU supports a fully pipelined $32 \times 32 \rightarrow 64$ bit integer multiplication, with a 2-cycle latency and a 1cycle inverse throughput. It is also possible to couple this multiplication with a 64-bit accumulation into a register pair at no additional cost.

The FPU, which shares its logic with the MAU, supports IEEE-754-compliant single-precision floating-point arithmetic, along with a few double-precision operations as well. However, we do not consider those in this work.

The LSU, in charge of all memory accesses, supports both 32- and 64-bit loads and stores. When the data is available in the cache, read instructions have a latency of only 2 cycles. A cache miss incurs a pipeline stall of approximately 10 cycles.

The BCU supports branches and function calls, which come at the cost of only a few cycles thanks to the low pipeline depth. The BCU also offers support for hardware loops, in which successive loop iterations are chained without any branching penalty.

Finally, since the Kalray-1 is a VLIW microarchitecture, it is possible to explicitly group instructions into instruction bundles which are to be issued at the same clock cycle and executed in parallel, as long as they are processed by different execution units. For instance, one can very well schedule in a single bundle a 64-bit addition (on the two ALUs), a 32bit multiplication (on the MAU), a 64-bit load (on the LSU), and a conditional branch (on the BCU). Even if this puts higher pressure on the compiler to extract parallelism from the code, this allows one to finely tune and optimize critical parts of an application at the assembly level.

\section{Multiprecision mOdULAR ARITHMETIC}

In this section, we present a flexible library for fast multiprecision modular arithmetic on the Kalray MPPA-256 processor. Even though $\mathrm{C}$ bindings are available for easy integration into larger projects, most of it is written in pure assembly code for efficiency purposes.

After detailing the data representation and algorithmic choices made in this library for the central operations, we present a few benchmark results in Section 3.8.

\subsection{Representation}

In the proposed library, integers are assumed to be unsigned (i.e., non-negative), and are represented in radix $2^{32}$ using arrays of 32 -bit words. For instance, the $n_{W}$-word array $\left(x_{0}, \ldots, x_{n_{W}-1}\right)$ represents the $\left(32 n_{W}\right)$-bit integer

$$
X=\sum_{i=0}^{n_{W}-1} x_{i} \cdot 2^{32 i}
$$

Picking a radix slightly smaller than the word size (such as $2^{30}$ ) would allow us to buffer carries instead of propagating them immediately in every arithmetic operation. However, the $\mathrm{K} 1$ instruction set offers carry-aware addition instructions at no extra cost, which render such considerations quite useless in the context of embarrassingly parallel applications such as ECM, in which no further parallelism needs to be leveraged from the arithmetic operations. Of course, should this not be the case-for instance in more memory-intensive applications, where higher-level parallelism cannot be achieved - using a redundant number system (such as carry-save, as above, or even RNS) to avoid carry propagations would prove key to distributing arithmetic computations over multiple threads.

In the usual context of ECM, the size of the integers $N$ we want to factor is known in advance. Consequently, for the sake of efficiency, the parameter $n_{W}$ is fixed at compile time using a preprocessor macro. Supported values for $n_{W}$ range from 2 to 16 , inclusive, which corresponds to moduli $N$ of size from 64 to 512 bits.

Note that, given the MPPA-256 two-level hierarchy of compute clusters and processing engines, it is perfectly possible to compile separate binaries with different values for $n_{W}$ and have them run simultaneously on distinct compute clusters. This would allow an ECM implementation to schedule incoming numbers $N$ on different clusters, according to their size, and even to dynamically reallocate compute resources to match the size distribution of these numbers. This is however not explored in this work.

\subsection{Basic integer operations}

Most of the basic arithmetic operations, such as integer addition, subtraction, comparison, assignment, and so on, were implemented in the proposed library. As can be expected, their time complexity $T_{\mathrm{op}}\left(n_{W}\right)$ is linear in $n_{W}$, and most of our optimization efforts concentrated on minimizing the ratio $T_{\mathrm{op}}\left(n_{W}\right) / n_{W}$. We illustrate this by detailing the case of the addition in the following paragraphs.

We suppose that we are given the address in memory of two $n_{W}$-word integers $X=\left(x_{0}, \ldots, x_{n_{W}-1}\right)$ and $Y=$ $\left(y_{0}, \ldots, y_{n_{W}-1}\right)$, and that we want to compute their sum as the $n_{W}$-word integer $R=\left(r_{0}, \ldots, r_{n_{W}-1}\right)$ along with the carry-out bit $c$ :

$$
X+Y=R+c \cdot 2^{32 n_{W}} .
$$


Since the K1 microarchitecture supports a 32-bit addwith-carry instruction (denoted by addc here) using a dedicated carry flag, a straightforward implementation would thus look something like the following pseudo-code (in which we denote by $X, Y$, and $R$ the registers containing the memory addresses of the corresponding multiprecision integers):

$\begin{array}{lr}\begin{array}{lr}\text { addc } 0,0 \\ i \leftarrow 0\end{array} & \text { (Clear carry flag) } \\ \text { repeat } n_{W} \text { times } & \text { (Initialize index) } \\ x \leftarrow \operatorname{load}[X+4 i] & \text { (Hardware loop) } \\ y \leftarrow \operatorname{load}[Y+4 i] & \text { (Load ith word } x_{i} \text { ) } \\ r \leftarrow \text { addc } x, y & \text { (Load ith word } y_{i} \text { ) } \\ {[R+4 i] \leftarrow \text { store } r} & \text { (Add with carry) } \\ i \leftarrow \text { add } i, 1 & \text { (Store ith word } r_{i} \text { ) } \\ c \leftarrow \text { addc } 0,0 & \text { (Increment index) }\end{array}$

Assuming the operands are already in the L1 cache, each load has a latency of 2 cycles. However, the two load's of each iteration can be pipelined and issued in two consecutive clock cycles. The add-with-carry, store, and increment instructions then require 1 cycle each, which gives a total of 6 cycles per iteration. Note that the use of a hardware loop allows us to completely avoid branching penalties after each iteration. We thus obtain a time complexity of $T_{\text {add }}\left(n_{W}\right)=6 n_{W}+O(1)$ cycles for the complete addition.

In fact, as mentioned earlier, the $\mathrm{K} 1$ instruction set includes 64-bit memory accesses, and the two main ALUs can be combined to support a 64-bit add-with-carry instruction. As these instructions have the same latency as their 32-bit counterparts, they can then be used to process the operands and compute the result two words at a time.

Furthermore, since the store and increment instructions are executed on different execution units (the LSU for the former, and one of the ALUs for the latter), both can be executed in parallel in the same clock cycle, thanks to the VLIW capabilities of the K1 microarchitecture, by explicitly writing these two instructions in the same instruction bundle at the assembly level.

These two improvements yield an addition having complexity $T_{\text {add }}\left(n_{W}\right)=5\left\lceil n_{W} / 2\right\rceil+O(1)$, as shown in the following pseudo-code (where the dotted horizontal lines delimitate the different instruction bundles and, for the sake of simplicity, restricted to the case where $n_{W}$ is even):

\begin{tabular}{|c|c|}
\hline $\mathrm{dec} 0,0$ & (Clear carry flag) \\
\hline$i \leftarrow$ & lize index) \\
\hline peat $n_{W} / 2$ times & re loop \\
\hline$x: x^{\prime} \leftarrow \operatorname{ioad}_{64}[X+8 i]$ & (Load ith dword) \\
\hline$y: y^{\prime} \leftarrow \operatorname{load}_{64}[Y+8 i]$ & (Load ith dword) \\
\hline$r: r^{\prime} \leftarrow \operatorname{addc}_{64} x: x^{\prime}, y: y^{\prime}$ & $(A d$ \\
\hline$[R+8 i] \leftarrow$ store $_{64} r: r^{\prime}$ & (Store $i t h d u$ \\
\hline$i \leftarrow$ add $i, 1$ & $(\operatorname{In}$ \\
\hline$\leftarrow \operatorname{addc} 0,0$ & (Save car \\
\hline
\end{tabular}

This is still not optimal, however: software pipelining techniques can be used to carefully rearrange and interleave the instructions of consecutive loop iterations, so as to maximize the instruction-level parallelism. For instance, one can schedule the addition-with-carry of the two $(i-1)$ st double-words $\left(x_{2 i-2}, x_{2 i-1}\right)$ and $\left(y_{2 i-2}, y_{2 i-1}\right)$ in parallel with the load of the next double-word $\left(x_{2 i}, x_{2 i+1}\right)$ :

$$
\begin{aligned}
& x: x^{\prime} \leftarrow \operatorname{load}_{64}[X] \text { (Load first dword) } \\
& \text { addc } 0,0 \quad \text { (Clear carry flag) } \\
& \left.y: y^{\prime} \leftarrow \text { load }_{64}[Y]\right]^{-} \text {(Load first dword) } \\
& i \leftarrow 1 \quad \text { (Initialize load index) } \\
& j \leftarrow 0 \quad \text { (Initialize store index) } \\
& \text { repeat } n_{W} / 2 \text { times } \\
& x: x^{\prime} \leftarrow \text { Ioad }_{64}[X+8 i] \quad \text { (Load ith dword) } \\
& r: r^{\prime} \leftarrow \operatorname{addc}_{64} x: x^{\prime}, y: y^{\prime} \quad \text { (Add with carry) } \\
& y: y^{\prime} \leftarrow \text { Ioad }_{64}[Y+8 i]^{\prime} \text { (Load ith dword) } \\
& i \leftarrow \text { add } i, 1 \quad \text { (Increment load index) } \\
& {[R+8 j] \leftarrow \text { store }_{64} r: r^{\prime} \text { (Store jth dword) }} \\
& j \leftarrow \text { add } j, 1 \quad \text { (Increment store index) } \\
& r: r^{\prime} \leftarrow \operatorname{addc}_{64} x: x^{\prime}, y: y^{\prime} \text { (Add with carry) } \\
& {[R+8 j] \leftarrow \text { store }_{64} r: r^{\prime} \cdot \text { (Store last dword) }} \\
& c \leftarrow \operatorname{addc} 0,0 \\
& \text { (Save carry flag) }
\end{aligned}
$$

The resulting instruction scheduling on the various execution units for two consecutive iterations of the loop is given in the following table. Instructions corresponding to the same double-words of the operands and of the result are shown in the same color.

\begin{tabular}{lll}
\hline Cycle & LSU & ALU $_{0} \& \mathrm{ALU}_{1}$ \\
\hline$\ldots$ & $\cdots$ & $\ldots$ \\
$t$ & $x: x^{\prime} \leftarrow \operatorname{load}_{64}[X+8 i]$ & $r: r^{\prime} \leftarrow \operatorname{addc}_{64} x: x^{\prime}, y: y^{\prime}$ \\
$t+1$ & $y: y^{\prime} \leftarrow \operatorname{lod}_{64}[Y+8 i]$ & $i \leftarrow \operatorname{add} i, 1$ \\
$t+2$ & {$[R+8 j] \leftarrow \operatorname{store}_{64} r: r^{\prime}$} & $j \leftarrow \operatorname{add} j, 1$ \\
$t+3$ & $x: x^{\prime} \leftarrow \operatorname{lod}_{64}[X+8 i]$ & $r: r^{\prime} \leftarrow \operatorname{addc} 64 x: x^{\prime}, y: y^{\prime}$ \\
$t+4$ & $y: y^{\prime} \leftarrow \operatorname{lod}_{64}[Y+8 i]$ & $i \leftarrow \operatorname{add} i, 1$ \\
$t+5$ & {$[R+8 j] \leftarrow \operatorname{store}_{64} r: r^{\prime}$} & $j \leftarrow$ add $j, 1$ \\
$\ldots$ & $\cdots$ & $\cdots$ \\
\hline
\end{tabular}

One can see from this scheduling that, even though the latency required to load, add, then store a pair of doublewords is 6 clock cycles, each iteration now has a latency of only 3 cycles. Therefore, the total time complexity for this operation is $T_{\text {add }}\left(n_{W}\right)=3\left\lceil n_{W} / 2\right\rceil+O(1)$ cycles.

This can be shown to be optimal, as the bottleneck for the addition lies in the Load/Store Unit, which has to load the $2\left\lceil n_{W} / 2\right\rceil$ double-words of the operands $X$ and $Y$, and store the $\left\lceil n_{W} / 2\right\rceil$ double-words of the result $R$, thus requiring at least $3\left\lceil n_{W} / 2\right\rceil$ clock cycles.

Finally, note that, when $n_{W}$ is small, a few cycles can be saved in the $O(1)$ part by fully unrolling the main loop. This avoids the constant-time overhead of the hardware loop, at the expense of an increase in code size, whose complexity jumps from $O(1)$ to $O\left(n_{W}\right)$.

\subsection{Basic modular arithmetic}

Basic modular operations such as negation, addition or subtraction directly rely on their integer counterparts on $n_{W^{-}}$ word operands described in the previous section. Operands are assumed to be already reduced with respect to the modulus $N$.

After the main operation, a final reduction step compares the result to the modulus $N$ and conditionally subtracts or adds it (in the case of a modular addition or subtraction, respectively). This comparison is performed most-significant digits first, so as to return an answer as quickly as possible. Thus, it has an average latency of only a few cycles, even though its worst-case complexity (in the case of equality) is still linear in $n_{W}$. 


\subsection{Integer multiplication}

Given two $n_{W}$-word multiprecision integers $X$ and $Y$, their $2 n_{W}$-word product $R=X \cdot Y$ is computed using a quadratic parallel-serial algorithm: the $n_{W}$ words of the multiplicand $X$ are first all loaded into registers, then, for $i$ ranging from 0 to $n_{W}-1$, each partial product $X \cdot y_{i}$ is computed, shifted left by $i$ words, and accumulated into the partial result:

$$
\begin{aligned}
& R \leftarrow 0 \\
& \text { for } i \leftarrow 0 \text { to } n_{W}-1 \text { do } \\
& \quad R \leftarrow R+X \cdot y_{i} \cdot 2^{32 i} \\
& \text { return } R
\end{aligned}
$$

Note that each partial product $X \cdot y_{i}$ fits on $n_{W}+1$ words, and that, before the $i$ th partial product is accumulated, the most-significant words $r_{n_{W}+i}$ to $r_{2 n_{W}-1}$ of the partial result are all 0 . Furthermore, because of the left shift by $i$ words, this means that the accumulation into $R$ will only modify words $r_{i}$ to $r_{n_{W}+i}$, and the carry need not be propagated further. Also, after accumulating the $i$ th partial product, the $i$ th word $r_{i}$ will have reached its final value, and may then be written back to memory. Consequently, at any point in the algorithm, only $n_{W}+1$ words of the partial result (from $r_{i}$ to $r_{n_{W}+i}$ ) need to be kept in the register file. Hence, the total number of registers required for the multiplication is $2 n_{W}+O(1)$.

In order to simplify the carry propagation when accumulating each partial product $X \cdot y_{i}$ into $R$, the words $x_{j}$ of the multiplicand $X$ are processed separately according to the parity of their index $j$ : we write $X=X_{0}+X_{1} \cdot 2^{32}$, with

$$
\begin{aligned}
& X_{0}=\sum_{\substack{k=0 \\
\left\lfloor n_{W} / 2\right\rfloor-1}}^{\left\lceil n_{W} / 2\right\rceil-1} x_{2 k} \cdot 2^{64 k}, \quad \text { and } \\
& X_{1}=\sum_{k=0} x_{2 k+1} \cdot 2^{64 k} .
\end{aligned}
$$

This way, we first compute the sub-product $S_{0}^{(i)}=X_{0} \cdot y_{i}$, whose individual products $x_{2 k} \cdot y_{i} \cdot 2^{64 k}$ are contiguous but do not overlap, and directly accumulate it into $R$. We then compute the second sub-product $S_{1}^{(i)}=X_{1} \cdot y_{i}$, which is also contiguous and overlap-free, and finally accumulate it into $R$ as well.

The Multiply-Accumulate Unit (MAU) of the K1 microarchitecture supports a $32 \times 32 \rightarrow 64$-bit integer multiplication, which has a latency of 2 cycles and an inverse throughput of 1 cycle, meaning that one such instruction can be issued at every clock cycle. As this matches the inverse throughput of the 64-bit add-with-carry instructions, we can therefore efficiently pipeline each individual product of $S_{0}^{(i)}$, and then of $S_{1}^{(i)}$, with its accumulation into $R$, using only two extra 64 -bit registers (denoted by $u: u^{\prime}$ and $v: v^{\prime}$ ) as buffers for the products.

The following scheduling illustrates this for the computation and accumulation of $S_{0}^{(i)}$ then of $S_{1}^{(i)}$ into $R$, for $n_{W}=8$, where we assume that the registers $x_{0}$ to $x_{n_{W}-1}$ contain the $n_{W}$ words of $X$, that $y$ contains $y_{i}$, and that $r_{0}$ to $r_{n_{W}}$ contain the $n_{W}+1$ "active" words $r_{i}$ to $r_{n_{W}+i}$ of the partial result:

\begin{tabular}{clc}
\hline Cycle & MAU & ALU $_{0} \& \mathrm{ALU}_{1}$ \\
\hline 0 & $u: u^{\prime} \leftarrow \operatorname{mul} x_{0}, y$ & \\
1 & $v: v^{\prime} \leftarrow \operatorname{mul} x_{2}, y$ & \\
2 & $u: u^{\prime} \leftarrow \operatorname{mul} x_{4}, y$ & $r_{0}: r_{1} \leftarrow \operatorname{addci}_{64} r_{0}: r_{1}, u: u^{\prime}$ \\
3 & $v: v^{\prime} \leftarrow \operatorname{mul} x_{6}, y$ & $r_{2}: r_{3} \leftarrow \operatorname{addc}_{64} r_{2}: r_{3}, v: v^{\prime}$ \\
4 & & $r_{4}: r_{5} \leftarrow \operatorname{addc}_{64} r_{4}: r_{5}, u: u^{\prime}$ \\
5 & $u: u^{\prime} \leftarrow \operatorname{mul} x_{1}, y$ & $r_{6}: r_{7} \leftarrow \operatorname{addc}_{64} r_{6}: r_{7}, v: v^{\prime}$ \\
6 & $v: v^{\prime} \leftarrow \operatorname{mul} x_{3}, y$ & $r_{8} \leftarrow \operatorname{addc}_{0} 0$ \\
7 & $u: u^{\prime} \leftarrow \operatorname{mul} x_{5}, y$ & $r_{0}: r_{1} \leftarrow \operatorname{addci}_{64} r_{1}: r_{2}, u: u^{\prime}$ \\
8 & $v: v^{\prime} \leftarrow \operatorname{mul} x_{7}, y$ & $r_{2}: r_{3} \leftarrow \operatorname{addc}_{64} r_{3}: r_{4}, v: v^{\prime}$ \\
9 & & $r_{4}: r_{5} \leftarrow \operatorname{addc}_{64} r_{5}: r_{6}, u: u^{\prime}$ \\
10 & & $r_{6}: r_{7} \leftarrow \operatorname{addc}_{64} r_{7}: r_{8}, v: v^{\prime}$
\end{tabular}

In the above scheduling, the addci $i_{64}$ instructions clear the carry flag before performing an addition-with-carry. This avoids having to use an extra instruction to do so. Also note that the indices of the output registers of the second sequence of addci $i_{64} / \operatorname{addc}_{64}$ 's are always one less than the indices of the corresponding input registers: this allows us to implement at no extra cost a sliding window for the $n_{W}+1$ "active" words of $R$, so that this pattern can be repeated in a loop to iterate through the words of $Y$. As a direct consequence, the register $r_{0}$ gets overwritten at cycle 7 : the contents of $r_{0}$ should therefore be stored back to memory as word $r_{i}$ between cycles 3 and 7 . Finally, one can verify that the final addition at cycle 10 will never generate an output carry.

We should also mention at this point that the K1 MAU supports a multiply-and-accumulate-with-carry instruction, which serves the same purpose as the combination of mul and $\operatorname{addc}_{64}$ we use here, only with a latency of only 2 cycles instead of 3 . However, this instruction has extra constraints regarding which pairs of 32-bit registers can be used as the accumulator: it turns out that these constraints are incompatible with the shift by one word that happens when accumulating $S_{1}^{(i)}$ into $R$ (see cycles 7 to 10 in the previous scheduling). This is why we decided not to use this instruction.

Hence, using this method, each partial product $X \cdot y_{i}$ can be computed and accumulated into $R$ in $n_{W}+3$ clock cycles. However, when iterating through the partial products, we can slightly overlap consecutive iteration by 2 cycles, thus reducing the cost to $n_{W}+1$ cycles per iteration, as depicted in the following "high-level" scheduling, for $n_{W}=8$, in which one can see the iteration pattern (highlighted here in a darker shade of gray, and delimited by dotted lines) repeating every 9 cycles.

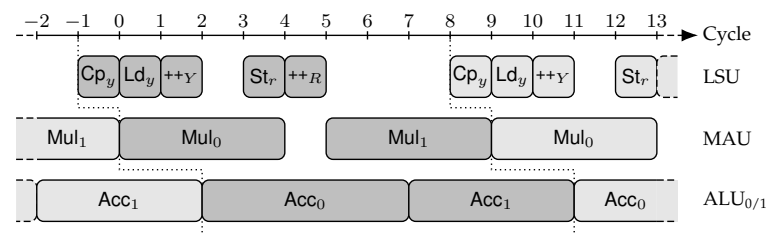

In this scheduling, the tasks $\mathrm{Mul}_{k}$ and $\mathrm{Acc}_{k}$ represent the computation and the accumulation of $S_{k}^{(i)}$, respectively. At each iteration, the multiplier word $y_{i}$, which was preloaded into a buffer register $y^{\prime}$ by the task $\operatorname{Ld}_{y}$ in the previous iteration, is copied into the actual register $y$ by task $\mathrm{Cp}_{y}$. Once computed, the result word $r_{i}$, contained in register $r_{0}$, is then stored back to memory by task $\mathrm{St}_{r}$. Finally, tasks $++_{Y}$ and $++_{R}$ are in charge of incrementing the read pointer on 
$Y$ and the write pointer on $R$, respectively.

One can show that this scheduling is optimal, as the two main ALUs have to accumulate and propagate carries through a total of $n_{W}+1$ words at each iteration (this would be also the case if the multiply-and-accumulate-with-carry instruction were used).

Therefore, all in all, our implementation computes a product of two $n_{W}$-word integers in $T_{\text {mul }}\left(n_{W}\right)=n_{W}\left(n_{W}+\right.$ 1) $+O(1)$ clock cycles, which is only slightly more than 1 cycle per individual word-by-word product.

Finally, note that subquadratic algorithms such as Karatsuba might be more efficient for larger values of $n_{W}$, but this is not the case for the sizes considered in this work, as the extra additions will induce a higher overhead than the saved multiplications. Even for $n_{W}=16$, according to Table 1, a single level of Karatsuba will require three 8-word multiplications, for a total cost of 243 cycles. This leaves only 44 cycles to perform the two 8-word additions and the three 16-word additions or subtractions and beat the 287 cycles required by the quadratic method. Even if we make the optimistic assumption that many of them can be merged with the prologue or the epilogue of the multiplications, it is very unlikely that the savings, if any, would be anything more than marginal.

This, however, warrants further investigation in order to find the precise crossover point.

\subsection{Squaring}

Currently, we do not have a specific implementation for squaring. We acknowledge this as a shortcoming. The two main advantages of the squaring situation are that the memory pressure due to the input is lower since there is only one input operand, and that the required number of word products is roughly halved. On the other hand, the structure of the code would be more complicated. For instance, it is almost certain that hardware loops can not be used. Also, the non-diagonal sub-products should be counted twice each, thus requiring a multiprecision leftshift by one bit before adding them to the diagonal subproducts. It is unclear how all of these could fit in the available computing units without too many bubbles in the pipelines. Furthermore, this might put back more pressure on the registers to store the whole product, while for the plain multiplication it can be written back to memory on the fly as only half of it needs to be kept in registers.

In our target application, during the stage 1 of ECM (see Section 4.3) about half of the multiplications are squarings. Being optimistic, we can hope for saving $25 \%$ on the cost of these. The modular reduction (see below) will be the same for multiplications and squarings, so that in the end, we can save roughly $25 \%$ on about one fourth of the total running time. To conclude, having specific code for squaring can certainly give some speed-up, but we do not expect more than a $10 \%$ saving on the total cost of the stage 1 of ECM. Furthermore, as far as the stage 2 (see Section 4.4) is concerned, since this step involves mostly multiplications with different operands, it would only marginally benefit from a faster squaring.

\subsection{Montgomery reduction}

Given an odd $n_{W}$-word modulus $N$ along with the constant $R=2^{32 n_{W}}$, the Montgomery reduction [13] of a $2 n_{W^{-}}$ word integer $X<N \cdot R$ with respect to $N$ is defined as $\operatorname{REDC}_{N}(X)=X \cdot R^{-1} \bmod N$. As $N<R$, using the Montgomery representation of integers modulo $N$, in which the elements $X \in \mathbb{Z} / N \mathbb{Z}$ are represented by $\widetilde{X}=X \cdot R \bmod N$, the product $Z=X \cdot Y \bmod N$ of two such residues $X$ and $Y \in \mathbb{Z} / N \mathbb{Z}$ can then be computed as $\widetilde{Z}=X \cdot Y \cdot R \bmod N=$ $\operatorname{REDC}_{N}(\tilde{X} \cdot \tilde{Y})$.

Given the precomputed constant $\widetilde{R}=R^{2} \bmod N$, conversions to and from this representation can be computed using only $n_{W}$-word integer multiplications and Montgomery reductions, as $\widetilde{X}=\operatorname{REDC}_{N}(X \cdot \widetilde{R})$ and $X=$ $\operatorname{REDC}_{N}(\widetilde{X})$, respectively.

Finally, as it is also compatible with addition, subtraction and negation modulo $N$, we can perform all the computations required for ECM in Montgomery representation in order to avoid conversions before and after each modular multiplication.

In [13], Montgomery gives an efficient algorithm requiring only multiplications for computing $\operatorname{REDC}_{N}(X)$, provided that the 1-word constant $n^{\prime}=(-N)^{-1} \bmod 2^{32}$ is known (thanks to a precomputation, for instance):

$$
\begin{aligned}
& T \leftarrow\left(x_{0}, \ldots, x_{n_{W}-1}\right) \quad \text { (i.e., } T \leftarrow X \bmod 2^{32 n_{W}} \text { ) } \\
& \text { for } i \leftarrow 0 \text { to } n_{W}-1 \text { do } \\
& q \leftarrow t_{0} \cdot n^{\prime} \bmod 2^{32} \\
& T \leftarrow x_{n_{W}+i} \cdot 2^{32\left(n_{W}-1\right)}+(T+q \cdot N) / 2^{32} \\
& \text { if } T \geq N \text { then } \\
& T \leftarrow T-N \\
& \text { return } T
\end{aligned}
$$

The partial result $T$ is first initialized with the $n_{W}$ least significant words of $X$. Then, at each iteration, a multiple of $N$ is added to it so as to make it divisible by $2^{32}$. The value of $T$ is then shifted right by one word, and the next word of $X$ is loaded and added (with carry) to $t_{n_{W}-1}$. A single final subtraction of $N$ might be necessary to keep the result below $N$.

At any point in the algorithm, $T$ is an $n_{W}$-word integer along with a delayed carry bit, and thus occupies $n_{W}+1$ registers denoted by $t_{0}$ to $t_{n_{W}}$. As the $n_{W}$-word modulus $N$ is also kept in the register file ( $n_{0}$ to $n_{n_{W}-1}$ ), the total number of registers required for this algorithm is then $2 n_{W}+O(1)$.

In fact, this algorithm is in many ways quite similar to that of the parallel-serial multiplication described in the previous section. In particular, by considering the odd- and even-indexed words of $N$ and by writing $N=N_{0}+N_{1} \cdot 2^{32}$ as we did for $X$ in the multiplication, we can also split the computation of the partial product $q \cdot N$ into two subproducts $S_{0}=q \cdot N_{0}$ and $S_{1}=q \cdot N_{1}$ and accumulate them separately into $T$. The only difference is that both accumulations into $T$ might generate output carries.

The proposed scheduling, which resembles that of the multiplication, thus requires two extra cycles to compute the quotient $q$ at the beginning of each iteration, and one extra cycle because of the longer carry chains. An example for $n_{W}=8$ words is given below. 


\begin{tabular}{ccc}
\hline Cycle & MAU & $\mathrm{ALU}_{0} \& \mathrm{ALU}_{1}$ \\
\hline 0 & $q \leftarrow \operatorname{mul} t_{0}, n^{\prime}$ & \\
1 & & \\
2 & $u: u^{\prime} \leftarrow \operatorname{mul} q, n_{0}$ & \\
3 & $v: v^{\prime} \leftarrow \operatorname{mul} q, n_{2}$ & \\
4 & $u: u^{\prime} \leftarrow \operatorname{mul} q, n_{4}$ & $0: t_{0} \leftarrow \operatorname{addci}_{64} t_{0}: t_{1}, u: u^{\prime}$ \\
5 & $v: v^{\prime} \leftarrow \operatorname{mul} q, n_{6}$ & $t_{1}: t_{2} \leftarrow \operatorname{addc}_{64} t_{2}: t_{3}, v: v^{\prime}$ \\
6 & $u: u^{\prime} \leftarrow \operatorname{mul} q, n_{1}$ & $t_{3}: t_{4} \leftarrow \operatorname{addc}_{64} t_{4}: t_{5}, u: u^{\prime}$ \\
7 & $v: v^{\prime} \leftarrow \operatorname{mul} q, n_{3}$ & $t_{5}: t_{6} \leftarrow \operatorname{addc}_{64} t_{6}: t_{7}, v: v^{\prime}$ \\
8 & $w: w^{\prime} \leftarrow \operatorname{mul} q, n_{5}$ & $t_{7}: t_{8} \leftarrow \operatorname{addc}_{64} t_{8}: 0, x: 0$ \\
9 & $u: u^{\prime} \leftarrow \operatorname{mul} q, n_{7}$ & $t_{0}: t_{1} \leftarrow \operatorname{addci}_{64} t_{0}: t_{1}, u: u^{\prime}$ \\
10 & & $t_{2}: t_{3} \leftarrow \operatorname{addc}_{64} t_{2}: t_{3}, v: v^{\prime}$ \\
11 & & $t_{4}: t_{5} \leftarrow \operatorname{addc}_{64} t_{4}: t_{5}, w: w^{\prime}$ \\
12 & & $t_{6}: t_{7} \leftarrow \operatorname{addc}_{64} t_{6}: t_{7}, u: u^{\prime}$ \\
13 & & $t_{8} \leftarrow \operatorname{addc}_{8}, 0$ \\
\hline
\end{tabular}

In the above scheduling, we assume that the current word $x_{n_{W}+i}$ of $X$ was loaded into register $x$ before cycle 8. Also note how the division of $T+q \cdot N$ by $2^{32}$ is handled transparently when accumulating $S_{0}$ into $T$ (cycles 4 to 8 ).

Even though each iteration takes $n_{W}+6$ cycles, we can overlap consecutive iterations by 4 cycles, resulting in an actual cost of $n_{W}+2$ cycles per iteration, as illustrated below in the case $n_{W}=8$ (in which $\mathrm{M}_{q}$ represents the computation of $q$ as $t_{0} \cdot n^{\prime}, \operatorname{Ld}_{x}$ and $+_{+}$the loading of $x_{n_{W}+i}$ followed by incrementing the corresponding pointer, and $\mathrm{Mul}_{k}$ and $\mathrm{Acc}_{k}$ the computation and accumulation of $S_{k}$, respectively):

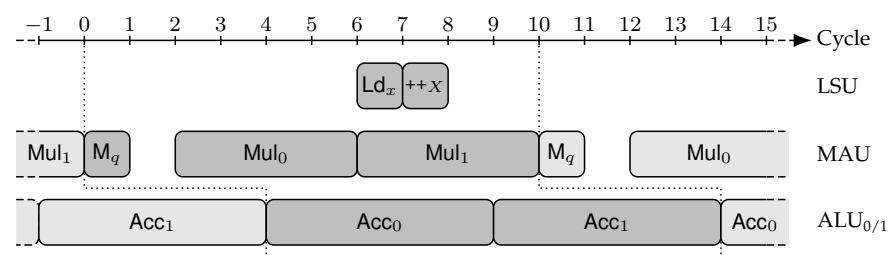

Therefore, the main loop of this algorithm requires $n_{W}\left(n_{W}+2\right)+O(1)$ cycles, to which we need to add $\left\lceil n_{W} / 2\right\rceil$ cycles for loading $N$ into the register file, and possibly another $\left\lceil n_{W} / 2\right\rceil$ cycles for subtracting $N$ from $T$. The comparison between $T$ and $N$ is assumed to have a constant average cost of a few cycles only. All in all, this gives a total average cost of $T_{\mathrm{REDC}}\left(n_{W}\right)=n_{W}\left(n_{W}+3\right)+O(1)$ clock cycles for the Montgomery reduction, just slightly above the cost of the integer multiplication.

Finally, as mentioned at the beginning of this section, the $\mathrm{REDC}_{N}$ function can be used to efficiently reduce a $2 n_{W}$-word product modulo $N$, and it is therefore called after each such multiplication. Variants of this Montgomery multiplication have been proposed where the computations of the product and of the reduction are interleaved [14], [15]. However, it turns out that our implementation would not benefit from such variants: the number of carry propagations to perform would change only marginally and, more importantly, the higher number of registers required would rapidly exhaust the register file and limit us to smaller values of $n_{W}$.

\subsection{GCD and modular inversion}

Our library also supports a few higher-level functions, which are implemented in C, on top of the low-level arithmetic primitives described previously. This is the case for a multiprecision GCD and for a multiprecision modular inversion (in Montgomery representation), as they are required in ECM. Both were implemented using the extended binary GCD algorithm.

\subsection{Benchmark results}

We report in Table 1 the latency of several functions of our multiprecision library, as measured for different operand sizes on the target MPPA-256 processor. These benchmarks assume that all data is already present in the L1 cache, so that no spurious cache-miss occurs. Due to the in-order nature of the $\mathrm{K} 1$ microarchitecture, these timings are extremely stable.

Note that almost all timings are given for fully unrolled versions of the low-level arithmetic functions (i.e., without hardware loops). The only low-level functions which were not unrolled are the integer multiplication and the Montgomery reduction for operand sizes above 256 bits $\left(n_{W}>8\right)$.

Timings for the modular functions (addition, Montgomery reduction and multiplication) are given as an interval, as the actual latency depends on whether final corrections (such as subtracting the modulus) have to be performed or not. However, these intervals do not include the worst-case latencies, which happen when the comparisons between the result and the modulus take linear time, as these occur only rarely.

Finally, timings for the GCD are given as the average for a hundred runs on random $n_{W}$-word inputs.

\section{The Elliptic Curve Method 4.1 Overview of the ECM algorithm}

There are many good descriptions of ECM in the literature [2] and we will not recall it in details, but we give here the general idea for completeness. Let $N$ be an integer to be tested for smoothness, and let $p$ be an (as-yet-unknown) prime factor of $N$. An elliptic curve $E$ defined over $\mathbb{Q}$ is chosen, together with a non-torsion point $P$ on $E$. We consider the reduction modulo $p$ of the point $P$, that is a point $\bar{P}$ that belongs to the reduced curve $\bar{E}$ over $\mathbb{Z} / p \mathbb{Z}$. Since the set of points on $\bar{E}$ is finite, $\bar{P}$ is of finite order $n_{\bar{P}}$ and, by Hasse-Weil's theorem, $n_{\bar{P}}$ is at most $p+1+2 \sqrt{p}$. If we can find a multiple $K$ of $n_{\bar{P}}$, then $\bar{Q}=K \cdot \bar{P}$ is the neutral element, namely the point at infinity of the reduced curve $\bar{E}$. In other words, $Q=K \cdot P$, as a point of the curve over the rationals, has coordinates whose common denominator $\delta$ is divisible by $p$. Hence the prime factor $p$ we are looking for divides the GCD of $\delta$ and $N$. And in general, unless $K$ is also a multiple of the order of the reduction of $P$ modulo another prime $q$ dividing $N$, we will have an equality $p=\operatorname{GCD}(\delta, N)$.

Since $p$ and therefore $n_{\bar{P}}$ are unknown, there is no efficient way to find for sure such a multiple $K$ of $n_{\bar{P}}$. ECM relies on the assumption that the group order $\# E(\mathbb{Z} / p \mathbb{Z})$ is a smooth number, so that $n_{\bar{P}}$ also has this property, and taking for $K$ the product of all the prime powers less than the smoothness bound, we get a multiple. Being smooth is not so rare a property, and after trying several different curves, one can usually retrieve the target prime factor of the integer $N$.

The general structure of the ECM algorithm for one curve is as follows. 
TABLE 1

Measured latencies (in clock cycles) of various functions for several operand sizes.

\begin{tabular}{lrrrrr}
\hline & & \multicolumn{3}{c}{ Latency according to operand sizes } \\
\cline { 3 - 6 } Function & Complexity & $\begin{array}{c}192 \text { bits } \\
\left(n_{W}=6\right)\end{array}$ & $\begin{array}{c}256 \text { bits } \\
\left(n_{W}=8\right)\end{array}$ & $\begin{array}{c}384 \text { bits } \\
\left(n_{W}=12\right)\end{array}$ & $\begin{array}{c}512 \text { bits } \\
\left(n_{W}=16\right)\end{array}$ \\
\hline Integer addition & $3 n_{W} / 2+O(1)$ & 16 & 19 & 25 & 31 \\
Integer multiplication & $n_{W}\left(n_{W}+1\right)+O(1)$ & 51 & 81 & 171 & 287 \\
\hline Modular addition & $9 n_{W} / 4+O(1)$ & $33-45$ & $36-51$ & $42-63$ & $48-75$ \\
Montgomery reduction & $n_{W}\left(n_{W}+3\right)+O(1)$ & $68-74$ & $95-102$ & $191-200$ & $314-325$ \\
Montgomery multiplication & $2 n_{W}\left(n_{W}+2\right)+O(1)$ & $121-127$ & $178-185$ & $364-373$ & $603-614$ \\
\hline GCD & $O\left(n_{W}{ }^{2}\right)$ & 12070 & 17745 & 30920 & 47560 \\
\hline Point addition (ext. coordinates) & $\mathbf{A}=8 \mathbf{m}+10 \mathbf{a}$ & 1321 & 1823 & 3402 & 5405 \\
Point addition (proj. coordinates) & $\mathbf{A}^{\prime}=7 \mathbf{m}+10 \mathbf{a}$ & 1205 & 1656 & 3044 & 4808 \\
Point doubling (ext. coordinates) & $\mathbf{D}=4 \mathbf{m}+4 \mathbf{s}+6 \mathbf{a}$ & 1184 & 1668 & 3212 & 5174 \\
Point doubling (proj. coordinates) & $\mathbf{D}^{\prime}=3 \mathbf{m}+4 \mathbf{s}+6 \mathbf{a}$ & 1061 & 1483 & 2841 & 4567 \\
\hline
\end{tabular}

1) Select a curve $E$ over $\mathbb{Q}$, together with a non-torsion point $P$;

2) Compute the point $Q=K \cdot P$, for $K=\prod_{\pi^{e} \leq B} \pi^{e}$, the product of prime powers less than $B$;

3) Take the GCD $g$ between the denominators of the coordinates of $Q$ and $N$;

4) If $g$ is a proper factor of $N$, return it. Otherwise, return FAIL.

By tuning the bound $B$ and the number of curves, it is possible to deduce an estimate of the probability that a number $N$ has no prime factor below a certain number of bits if ECM failed repeatedly.

An important remark is that Step 3 requires only to know the coordinates of $Q$ modulo $N$, and therefore the computation of Step 2 can and must be done modulo $N$ and not over the rationals, so that the size of the coordinates remains bounded. Then, of course, some inversions might fail because $N$ is not a prime, but then, the corresponding Extended GCD computation will readily reveal a proper factor of $N$ that would have been found at Step 3. An alternative is to use projective coordinates so that no inversion occur (since they are expensive); then the "denominator" used in Step 3 is just the third component of the projective coordinates.

Usually, Step 2 is decomposed into two stages, corresponding to a refinement of the notion of smoothness. Two bounds $B_{1}<B_{2}$ are chosen, and we assume that the order $n_{\bar{P}}$ has all its prime factors below the bound $B_{1}$ except for perhaps one factor that must still be below the bound $B_{2}$. This 2-bound smoothness notion is stronger than just $B_{1}$-smoothness, but less general than $B_{2}$-smoothness. The advantage is that it is much cheaper to test for 2bound smoothness than for the true $B_{2}$-smoothness. Our implementation follows this 2-stage strategy.

\subsection{Curve arithmetic}

Most of the time is spent in the elliptic curve group law, where coordinates are integers modulo $N$. Hence having fast modular arithmetic is crucial for efficiency. At a higher level, it is important to choose an appropriate coordinate system for the elliptic curve, reducing the number of operations in $\mathbb{Z} / N \mathbb{Z}$, and also appropriate addition chains to reduce the number of additions and doublings on the elliptic curve. Since the Kalray MMPA-256 processor has similar characteristics as GPUs-namely, a lot of computing power but limited or slow memory access-we followed the same strategy as the one used in the state-of-the-art implementations of ECM on GPUs [8], [9]. Therefore, we used the so-called extended coordinates on twisted Edwards curves with $a=-1$ [16], [17].

The costs in terms of modular multiplications (m), squarings (s) and additions / subtractions (a) of point addition (A) and doubling (D) in this coordinate system are recalled in Table 1, along with average latencies benchmarked for various sizes of the modulus $N$. When only projective coordinates are required for the result, a modular multiplication can be saved in both operations (which are then denoted by $\mathbf{A}^{\prime}$ and $\mathbf{D}^{\prime}$, respectively).

Classically, we used a two-stage scalar multiplication, where stage 1 is performed using no-storage addition chains as developed in [8], while stage 2 relies on a babystep/giant-step approach, again following [9]. In the following, we give a few more details on these two stages, since we slightly modified them compared to [8], [9].

\subsection{Addition chains for stage 1}

The textbook stage 1 of ECM consist in multiplying $P$ by a scalar of the form $\prod_{\pi^{e} \leq B_{1}} \pi^{e}$ for a given bound $B_{1}$. The idea of [8] is to group primes $\pi$ occurring in this product in batches having low Hamming-weight, so that a scalar multiplication by those batches involves less additions than we would have with the original scalar. Finding the best chains based on this idea would imply a fully exponential search; however, using a massive precomputation it is still possible to find very good chains with a simple greedy heuristic.

We have implemented the method presented in [8] and searched for no-storage addition chains, only with a slight modification of the initial ordering of the available addition 
chains $s_{i}$ : instead of using the ratio $r\left(s_{i}\right)=\mathrm{dbl}\left(s_{i}\right) / \operatorname{add}\left(s_{i}\right)$ as in $[8$, Algorithm 1], we used the quantity

$$
\kappa\left(s_{i}\right)=\frac{\log _{2}\left(s_{i}\right)}{\operatorname{dbl}\left(s_{i}\right)+(8 / 7) \cdot \operatorname{add}\left(s_{i}\right)-\log _{2}\left(s_{i}\right)},
$$

where the constant $8 / 7$ comes from the approximate cost ratio between an addition in extended coordinates $(\mathbf{A} \approx 8 \mathbf{m})$ and a doubling in projective coordinates $\left(\mathbf{D}^{\prime} \approx 3 \mathbf{m}+4 \mathbf{s}\right)$.

We chose this metric as it better takes into account the number of bits actually contributed by each addition chain. For instance, while $r(1665)=r(863)=10 / 3$, as both chains can be computed in 10 doublings and 3 additions, we have $\kappa(1665) \approx 3.92$ and $\kappa(863) \approx 2.65$, as the former is almost the double of the latter.

The addition chains we found matched the results of [8], only with a very minor improvement. Their costs are given in Table 2 for various values of $B_{1}$, along with the corresponding timings.

\subsection{Stage 2 based on baby-step/giant-step}

The idea of the stage 2 strategy is to test, for all primes $\pi$ between $B_{1}$ and another bound $B_{2}$, whether $\pi$ times the point $Q$ coming out from stage 1 is the neutral element. This is achieved in a batch way, where the number of curve operations grows only like the square root of $B_{2}-B_{1}$. Let $w$ be the value taken for the giant-steps: we write all the primes $B_{1}<\pi \leq B_{2}$ as $\pi=v w \pm u$, where

$$
\begin{aligned}
& u \in U=\left\{u \in \mathbb{Z} \mid 1 \leq u \leq \frac{w}{2}, \operatorname{gcd}(u, w)=1\right\}, \text { and } \\
& v \in V=\left\{v \in \mathbb{Z} \mid\left\lceil\frac{B_{1}}{w}-\frac{1}{2}\right\rceil \leq v \leq\left\lfloor\frac{B_{2}}{w}+\frac{1}{2}\right\rfloor\right\} .
\end{aligned}
$$

The algorithm then computes all the points $[u] Q$ and $[v w] Q$ for $u \in U$ and $v \in V$. Finally, by constructing appropriate products of scalar based on the coordinates of these points, it is possible to test whether one among all the points is indeed the neutral element with only one GCD with $N$. This final construction is very similar to the one used in Montgomery's batch inversion, and we refer to [9], [18] for details.

In this setting, it is interesting to take for $w$ a smooth number so that the set $U$ has a small number of elements, which reduces the running time and the memory storage. Furthermore, this number should be around the square root of $B_{2}-B_{1}$. In [9], they choose $B_{2}=16384$, and $w=2$. $3 \cdot 5 \cdot 7=210$. However, we found that, for this value of $B_{2}$, it is better to choose $w=420$, yielding a total cost of 2538 multiplications in $\mathbb{Z} / N \mathbb{Z}$ instead of 2690 with $w=210$. Similarly, when $B_{2}$ increases, it is better to choose larger multiples of 210 for $w$.

Choices of $w$ for several values of $B_{1}$ and $B_{2}$ are given in Table 3, along with the corresponding costs and timings.

\subsection{Benchmark results}

In Tables 2 and 3, we report the number of operations and the measured latency for the two stages of ECM, for a few typical modulus sizes and $B_{1}, B_{2}$ parameters. These benchmarks were run on a single core of a single cluster, so that all the required data fit easily in memory.
For the stage 1, the measured latencies include the cost of a final GCD. This operation and the numerous additions account for the difference observed between the latency of the full stage 1 and the naive estimate obtained by multiplying the number of modular multiplications by the latency of a single modular multiplication as reported in Table 1. According to our measures there seems to be no other significant overhead for the stage 1.

For the stage 2, the reported latencies also include a final GCD. For each $B_{1}$, the value of $B_{2}$ has been chosen as an integer multiple of $2^{14}$ such that the number of multiplications required is about the same as in the stage 1 . This step is however more memory intensive. This becomes particularly visible in the last two lines of the table. For instance, in the case of $B_{1}=8192$ and $B_{2}=80 \cdot 2^{14}$, the arithmetic cost of the stage 2 is very similar to that of the stage 1 (around $90 \mathrm{k}$ multiplications and as many additions for each stage). However, the measured latency of the stage 2 is about $10 \%$ higher than that of the stage 1 . We interpret this as the cost of the cache-misses that must be more frequent with such large values of $B_{2}$.

In Table 4, we finally provide benchmarks that are close to what we would have in an NFS context, during the cofactorization step of the relation collection, or during the initialization of a discrete logarithm descent (for the large modulus sizes and values of $B_{1}$ and $B_{2}$ ). The 256 cores of the processor are working in parallel, each core working independently of the others on a particular modulus. The benchmark also includes the time for the data transfer between the I/O subsystems and the compute clusters. The costs for the initialization of the curve and the Montgomery constants for the given modulus are included as well. Not much effort has been put in optimizing these functionalities, and this explains the overhead of about $20 \%$ for the smallest cases ( $B_{1}=256$ for 192- and 256-bit moduli) compared to what we would expect by just taking the latencies of Tables 2 and 3 and deducing a lower bound for the throughput. For all the other cases, the overhead compared to the lower bound remains below $10 \%$. For the largest examples that require a lot of memory, the 16 cores of each cluster are divided into 8 pairs: in each pair, the first core only does stage 1's while the second one only does stage 2's. Since the parameters were chosen so that the two stages take about the same time, we can pipeline a modulus through the two cores of a pair while keeping the additional overhead due to thread synchronization quite low.

During these full benchmarks, the average power consumption reported by the monitoring tools of the MPPA-256 card was $16 \mathrm{~W}$. The "throughput per joule" estimates given in Table 4 are based on this value.

\subsection{Comparison with other ECM implementations}

We have compared our implementation with the ones previously reported in the literature, using two criteria: the number of curves processed per second and the number of curves per joule. Since there is no official price for the MPPA-256 processor, comparisons based on curves per dollar, as done in some articles, were not possible. The results are given in Table 5. For comparing to general-purpose hardware, we used the EECM-MPFQ software which is 
TABLE 2

Cost and measured latencies (in clock cycles) for the stage 1 of ECM.

\begin{tabular}{|c|c|c|c|c|c|c|c|}
\hline \multirow[b]{2}{*}{$B_{1}$} & \multicolumn{3}{|l|}{ Cost } & \multicolumn{4}{|c|}{ Average latency according to size of modulus $N$} \\
\hline & $\begin{array}{l}\text { Number of operations } \\
\text { (curve ops. and mults.) }\end{array}$ & $\begin{array}{c}\text { Total \# } \\
\text { of mults. }\end{array}$ & $\begin{array}{l}\text { Difference } \\
\text { with [8] }\end{array}$ & $\begin{array}{l}192 \text { bits } \\
\left(n_{W}=6\right)\end{array}$ & $\begin{array}{c}256 \text { bits } \\
\left(n_{W}=8\right)\end{array}$ & $\begin{array}{c}384 \text { bits } \\
\left(n_{W}=12\right)\end{array}$ & $\begin{array}{c}512 \text { bits } \\
\left(n_{W}=16\right)\end{array}$ \\
\hline 256 & $38 \mathbf{A}+12 \mathbf{m}=$ & $2843 \mathrm{~m}$ & $-1 \mathrm{~m}$ & $444 \mathrm{k}$ & $621 \mathrm{k}$ & $1.18 \mathrm{M}$ & $1.90 \mathrm{M}$ \\
\hline 512 & $74 \mathbf{A}+21 \mathbf{m}=$ & $5786 \mathbf{m}$ & $-20 \mathbf{m}$ & $894 \mathrm{k}$ & $1.25 \mathrm{M}$ & $2.39 \mathrm{M}$ & $3.83 \mathrm{M}$ \\
\hline 1024 & $1473 \mathbf{D}^{\prime}+140 \mathbf{A}+37 \mathbf{m}=$ & $=11468 \mathbf{m}$ & $-40 \mathrm{~m}$ & $1.77 \mathrm{M}$ & $2.47 \mathrm{M}$ & $4.71 \mathrm{M}$ & $7.55 \mathrm{M}$ \\
\hline 8192 & $11774 \mathbf{D}^{\prime}+1015 \mathbf{A}+192 \mathbf{m}=$ & $=90730 \mathbf{m}$ & $-344 \mathbf{m}$ & $13.9 \mathrm{M}$ & $19.4 \mathrm{M}$ & $37.1 \mathrm{M}$ & $59.6 \mathrm{M}$ \\
\hline 32768 & $47158 \mathbf{D}^{\prime}+3899 \mathbf{A}+647 \mathbf{m}=$ & $=361945 \mathbf{m}$ & - & $55.3 \mathrm{M}$ & $77.5 \mathrm{M}$ & $148 \mathrm{M}$ & $237 \mathrm{M}$ \\
\hline
\end{tabular}

TABLE 3

Cost and measured latencies (in clock cycles) for the stage 2 of ECM.

\begin{tabular}{|c|c|c|c|c|c|c|c|c|}
\hline \multirow[b]{2}{*}{$B_{1}$} & \multirow[b]{2}{*}{$B_{2}$} & \multirow[b]{2}{*}{$w$} & \multicolumn{2}{|l|}{ Cost } & \multicolumn{4}{|c|}{ Average latency according to size of modulus $N$} \\
\hline & & & $\begin{array}{l}\text { Number of operations } \\
\text { (curve ops. and mults.) }\end{array}$ & $\begin{array}{l}\text { Total \# } \\
\text { of mults. }\end{array}$ & $\begin{array}{l}192 \text { bits } \\
\left(n_{W}=6\right)\end{array}$ & $\begin{array}{l}256 \text { bits } \\
\left(n_{W}=8\right)\end{array}$ & $\begin{array}{c}384 \text { bits } \\
\left(n_{W}=12\right)\end{array}$ & $\begin{array}{c}512 \text { bits } \\
\left(n_{W}=16\right)\end{array}$ \\
\hline 256 & $2^{14}$ & $2 \cdot 210$ & $1802 \mathbf{m}=$ & $2538 \mathrm{~m}$ & $400 \mathrm{k}$ & $561 \mathrm{k}$ & $1.07 \mathrm{M}$ & $1.72 \mathrm{M}$ \\
\hline 512 & $3 \cdot 2^{14}$ & $3 \cdot 210$ & $43 \mathbf{D}+112 \mathbf{A}+$ & $5812 \mathbf{m}$ & $913 \mathrm{k}$ & $1.28 \mathrm{M}$ & $2.44 \mathrm{M}$ & $3.93 \mathrm{M}$ \\
\hline 1024 & $7 \cdot 2^{14}$ & $5 \cdot 210$ & $58 \mathbf{D}+176 \mathbf{A}+$ & $=11410 \mathrm{~m}$ & $1.80 \mathrm{M}$ & $2.52 \mathrm{M}$ & $4.79 \mathrm{M}$ & $7.71 \mathrm{M}$ \\
\hline 8192 & $80 \cdot 2^{14}$ & $22 \cdot 210$ & $147 \mathbf{D}+624 \mathbf{A}+84954 \mathbf{m}=$ & $=91122 \mathbf{m}$ & $15.4 \mathrm{M}$ & $21.1 \mathrm{M}$ & $40.1 \mathrm{M}$ & $64.1 \mathrm{M}$ \\
\hline 32768 & $360 \cdot 2^{14}$ & $33 \cdot 210$ & $430 \mathbf{D}+1148 \mathbf{A}+343716 \mathbf{m}=$ & $=356340 \mathbf{m}$ & $61.8 \mathrm{M}$ & $83.7 \mathrm{M}$ & $158 \mathrm{M}$ & $252 \mathrm{M}$ \\
\hline
\end{tabular}

TABLE 4

Measured throughput (in curves per second and curves per joule) for the full implementation of ECM.

\begin{tabular}{|c|c|c|c|c|c|c|c|c|c|}
\hline \multirow[b]{2}{*}{$B_{1}$} & \multirow[b]{2}{*}{$B_{2}$} & \multicolumn{8}{|c|}{ Average number of curves per second and per joule according to size of modulus $N$} \\
\hline & & \multicolumn{2}{|c|}{192 bits $\left(n_{W}=6\right)$} & \multicolumn{2}{|c|}{256 bits $\left(n_{W}=8\right)$} & \multicolumn{2}{|c|}{384 bits $\left(n_{W}=12\right)$} & \multicolumn{2}{|c|}{512 bits $\left(n_{W}=16\right)$} \\
\hline 256 & $2^{14}$ & $105 \mathrm{k} / \mathrm{s}$ & $6.56 \mathrm{k} / \mathrm{J}$ & $76.6 \mathrm{k} / \mathrm{s}$ & $4.79 \mathrm{k} / \mathrm{J}$ & $41.4 \mathrm{k} / \mathrm{s}$ & $2.59 \mathrm{k} / \mathrm{J}$ & $25.9 \mathrm{k} / \mathrm{s}$ & $1.62 \mathrm{k} / \mathrm{J}$ \\
\hline 512 & $3 \cdot 2^{14}$ & $52.9 \mathrm{k} / \mathrm{s}$ & $3.31 \mathrm{k} / \mathrm{J}$ & $38.1 \mathrm{k} / \mathrm{s}$ & $2.38 \mathrm{k} / \mathrm{J}$ & $20.2 \mathrm{k} / \mathrm{s}$ & $1.26 \mathrm{k} / \mathrm{J}$ & $12.6 \mathrm{k} / \mathrm{s}$ & 788 /J \\
\hline 1024 & $7 \cdot 2^{14}$ & $27.6 \mathrm{k} / \mathrm{s}$ & $1.73 \mathrm{k} / \mathrm{J}$ & $19.9 \mathrm{k} / \mathrm{s}$ & $1.24 \mathrm{k} / \mathrm{J}$ & $10.5 \mathrm{k} / \mathrm{s}$ & $656 / \mathrm{J}$ & $6.53 \mathrm{k} / \mathrm{s}$ & $408 / \mathrm{J}$ \\
\hline 8192 & $80 \cdot 2^{14}$ & $3.49 \mathrm{k} / \mathrm{s}$ & $218 / \mathrm{J}$ & $2.47 \mathrm{k} / \mathrm{s}$ & $154 / \mathrm{J}$ & $1.22 \mathrm{k} / \mathrm{s}$ & $76.3 / \mathrm{J}$ & $761 / \mathrm{s}$ & $47.6 / \mathrm{J}$ \\
\hline 32768 & $360 \cdot 2^{14}$ & $795 / \mathrm{s}$ & $49.7 / \mathrm{J}$ & $572 / \mathrm{s}$ & $35.8 / \mathrm{J}$ & & & & \\
\hline
\end{tabular}

an adaptation of GMP-ECM targetting specially the sizes considered in the present article. This experiment was run on a machine with two Intel E5-2650 processors, each having 8 cores, with an announced TDP of $95 \mathrm{~W}$ each. Thanks to hyperthreading, the best throughput was obtained by running 32 threads in parallel. Due to a different stage 2 strategy, it was not possible to obtain exactly the same value of $B_{2}$ as in our implementation, so we set the parameters to get a close enough value.

For GPU-based implementations, we did not run the experiments ourselves but copied the data given in [8], [9] which are the best published results so far for ECM on graphics cards. The implementation of [8] contains only a stage 1, so we extrapolated the throughput of our implementation for such a setting using the data of the line $B_{1}=1024$ and $B_{1}=8192$ of Table 2 . This is not very precise but is anyway considered rather obsolete since a stage 2 implementation finds many more prime factors.

From the results in Table 5, it is clear that the generalpurpose processors are not well suited: even in terms of pure throughput, modern Intel processors can hardly compete with the MPPA-256 processor, and if the power consumption is taken into account, they are far behind.

The comparison with the GPU implementation is more balanced: a single GPU chip can process 2 to 3 times as many curves per second, depending on the size of the modulus. On the other hand it requires a lot of energy and, in terms of curves per joule, the advantage is clearly on the MPPA-256 side. It must also be noted that our implementation is much more versatile: it is possible to handle much larger $B_{1}, B_{2}$ and sizes of moduli with only a moderate penalty.

It should be noted that our ECM implementation on the MPPA-256 processor, in terms of energy efficiency, promises to outperform even results with recent GPUs, such as the NVIDIA GeForce GTX TITAN X (launched in 2015) which is the most powerful graphic card manufactured with $28 \mathrm{~nm}$ processors. The peak power consumption of GTX TITAN $X$ is $250 \mathrm{~W}$, which is slightly higher than that of GTX580, while the processor performance in single precision FLOPS is about 4 times higher. Thus, even if we implemented ECM on such recent GPUs following [9], the resulting throughput should still fall behind our MPPA-256 implementation in 
terms of curves per joule.

Finally, note that the curve-per-joule throughputs for CPUs and GPUs were obtained using the claimed TDP for those chips (which is probably an overestimation), while the MPPA benchmarks come from actual power measurements that we obtained using the monitoring facilities of the development board.

However, even if we assume that the actual power consuption of the CPUs and GPUs during these benchmarks was 2 or 3 times lower than their TDP, the MPPA-256 implementation would still be ahead.

\section{Conclusion}

In this article we have shown how to implement a multiprecision modular arithmetic library for the Kalray MPPA256 processor for moduli of up to 512 bits, where quadratic multiplication algorithms are well suited. The architecture of the processing engines (the cores) at the heart of this processor proved to be convenient for the task, since in our implementation, the pipelines of all the main execution units remain always busy: no obvious bottleneck could be found that would penalize the efficiency.

On top of this library, we have implemented the ECM algorithm for factoring integers with parameters that are useful for its application in the Number Field Sieve. In this setting, the latency is not an issue and the throughput is the main criterion for comparison. The results are quite satisfactory, with a throughput obtained with the Kalray MPPA-256 processor that is only slightly smaller than for a graphics card, but with a much lower power consumption. Also, the amount of fast memory available for each core is large enough to handle sizes that were not reachable in graphics cards.

\section{REFERENCES}

[1] H. W. Lenstra, Jr., "Factoring integers with elliptic curves," Annals of Mathematics, vol. 126, no. 3, pp. 649-673, 1987.

[2] P. Zimmermann and B. Dodson, "20 years of ECM," in ANTS VII, ser. LNCS, F. Hess, S. Pauli, and M. Pohst, Eds., vol. 4076. Springer, 2006, pp. 525-542.

[3] D. J. Bernstein, P. Birkner, T. Lange, and C. Peters, "ECM using Edwards curves," Mathematics of Computation, vol. 82, no. 282, pp. 1139-1179, 2013.

[4] A. K. Lenstra and H. W. Lenstra, Jr., Eds., The development of the Number Field Sieve, ser. Lecture Notes in Mathematics. Springer, 1993, vol. 1554.

[5] D. Coppersmith, "Modifications to the Number Field Sieve," Journal of Cryptology, vol. 6, no. 3, pp. 169-180, 1993.

[6] D. J. Bernstein and T. Lange, "Batch NFS," in $S A C^{\prime} 14$, ser. LNCS, A. Joux and A. Youssef, Eds., vol. 8781. Springer, 2014, pp. 38-58.

[7] D. Adrian, K. Bhargavan, Z. Durumeric, P. Gaudry, M. Green, J. A. Halderman, N. Heninger, D. Springall, E. Thomé, L. Valenta, B. VanderSloot, E. Wustrow, S. Zanella-Béguelin, and P. Zimmermann, "Imperfect forward secrecy: How Diffie-Hellman fails in practice," in CCS'15, I. Ray, N. Li, and C. Kruegel, Eds. ACM, 2015, pp. 5-17.

[8] J. W. Bos and T. Kleinjung, "ECM at work," in ASIACRYPT'12, ser. LNCS, X. Wang and K. Sako, Eds., vol. 7658. Springer, 2012, pp. 467-484.

[9] A. Miele, J. W. Bos, T. Kleinjung, and A. K. Lenstra, "Cofactorization on graphics processing units," in CHES'14, ser. LNCS, L. Batina and M. Robshaw, Eds., vol. 8731. Springer, 2014, pp. 335-352.

[10] "Kalray." [Online]. Available: http://www.kalray.eu
[11] B. Dupont de Dinechin, P. Guironnet de Massas, G. Lager, C. Léger, B. Orgogozo, J. Reybert, and T. Strudel, "A distributed runtime environment for the Kalray MPPA-256 integrated manycore processor," in ICCS'13, ser. Procedia Computer Science, V. Alexandrov, M. Lees, V. Krzhizhanovskaya, J. Dongarra, and P. M. A. Sloot, Eds., vol. 18. Elsevier, 2013, pp. 1654-1663.

[12] B. Dupont de Dinechin, R. Ayrignac, P.-E. Beaucamps, P. Couvert, B. Ganne, P. Guironnet de Massas, F. Jacquet, S. Jones, N. Morey Chaisemartin, F. Riss, and T. Strudel, "A clustered manycore processor architecture for embedded and accelerated applications," in HPEC'13. IEEE, 2013.

[13] P. L. Montgomery, "Modular multiplication without trial division," Mathematics of Computation, vol. 44, no. 170, pp. 519-521, 1985.

[14] S. R. Dussé and B. S. Kaliski, Jr., "A cryptographic library for the Motorola DSP56000," in EUROCRYPT'90, ser. LNCS, I. B. Damgård, Ed., vol. 473. S Springer, 1991, pp. 230-244.

[15] C.. K. Koç, T. Acar, and B. S. Kaliski, Jr., "Analyzing and comparing Montgomery multiplication algorithms," IEEE Micro, vol. 16, no. 3, pp. 26-33, 1996.

[16] D. J. Bernstein, P. Birkner, M. Joye, T. Lange, and C. Peters, "Twisted Edwards curves," in AFRICACRYPT'08, ser. LNCS, S. Vaudenay, Ed., vol. 5023. Springer, 2008, pp. 389-405.

[17] H. Hisil, K. K.-H. Wong, G. Carter, and E. Dawson, "Twisted Edwards curves revisited," in ASIACRYPT'08, ser. LNCS, J. Pieprzyk, Ed., vol. 5350. Springer, 2008, pp. 326-343.

[18] P. L. Montgomery, "Speeding the Pollard and elliptic curve methods of factorization," Mathematics of Computation, vol. 48, no. 177, pp. 243-264, 1987.

[19] D. J. Bernstein, P. Birkner, T. Lange, and C. Peters, "EECM: ECM using Edwards curves," 2010, software. [Online]. Available: http:/ /eecm.cr.yp.to/

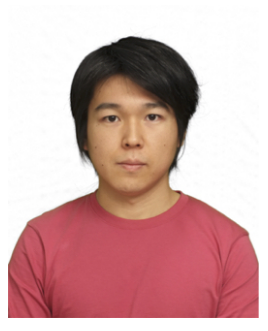

Masahiro Ishii received M.S. degree from Nagoya University in 2011 and received M.E. and his Ph.D. in engineering from Nara Institute of Science and Technology in 2013 and 2016, respectively. He is currently a specially appointed assistant professor at Tokyo Institute of Technology. His research interests are pairing-based cryptography and (hyper)elliptic curve cryptography, and efficient software implementation including GPU programming for cryptographic applications.

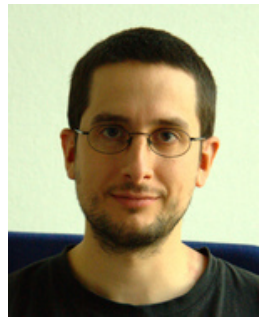

Jérémie Detrey received his M.Sc. and his Ph.D. in computer science in the Arénaire team at LIP, ÉNS Lyon (France) in 2003 and 2007 respectively. He is now a junior researcher at INRIA Nancy - Grand Est, and a member of the CARAMBA team at LORIA in Nancy (France). His research interests cover the various implementation-related aspects of computer arithmetic, with a special focus on finite fields, elliptic curves, and their applications to cryptography.

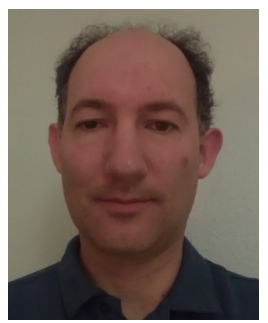

Pierrick Gaudry received his Ph.D. in computer science at the École Polytechniqe (France) in 2000. He is a CNRS researcher in the CARAMBA team at LORIA in Nancy (France). His research interests are mainly in algorithmic number theory in relation to cryptography, with an emphasize on elliptic curves, integer factorization and the discrete logarithm problem. 
TABLE 5

Comparison with other ECM implementations for various parameters, in curves per second and curves per joule.

\begin{tabular}{|c|c|c|c|c|c|c|c|}
\hline $\begin{array}{l}\text { Stage } 1 \text { only } \\
\text { Platform [ref] }\end{array}$ & $B_{1}$ & & Size of $N$ & $\begin{array}{l}\text { Curves } \\
\text { per second }\end{array}$ & $\begin{array}{l}\text { Ratio wrt. } \\
\text { this work }\end{array}$ & $\begin{array}{l}\text { Curves } \\
\text { per joule }\end{array}$ & $\begin{array}{l}\text { Ratio wrt. } \\
\text { this work }\end{array}$ \\
\hline \multirow[t]{2}{*}{ GTX580 [8] } & 960 & & 192 bits & $171 \mathrm{k} / \mathrm{s}$ & 2.96 & $702 / \mathrm{J}$ & 0.19 \\
\hline & 8192 & & 192 bits & $19.9 \mathrm{k} / \mathrm{s}$ & 2.70 & $81 / \mathrm{J}$ & 0.18 \\
\hline $\begin{array}{l}\text { Stage } 1 \text { and stage } 2 \\
\text { Platform [ref] }\end{array}$ & $B_{1}$ & $B_{2}$ & Size of $N$ & $\begin{array}{c}\text { Curves } \\
\text { per second }\end{array}$ & $\begin{array}{l}\text { Ratio wrt. } \\
\text { this work }\end{array}$ & $\begin{array}{l}\text { Curves } \\
\text { per joule }\end{array}$ & $\begin{array}{l}\text { Ratio wrt. } \\
\text { this work }\end{array}$ \\
\hline \multirow[t]{3}{*}{ GTX580 [9] } & 256 & $2^{14}$ & 192 bits & $309 \mathrm{k} / \mathrm{s}$ & 2.94 & $1.27 \mathrm{k} / \mathrm{J}$ & 0.19 \\
\hline & & & 256 bits & $180 \mathrm{k} / \mathrm{s}$ & 2.35 & 738 /J & 0.15 \\
\hline & & & 384 bits & $86 \mathrm{k} / \mathrm{s}$ & 2.08 & $352 / \mathrm{J}$ & 0.14 \\
\hline \multirow{14}{*}{$\begin{array}{l}\text { EECM-MPFQ [19] } \\
\text { (dual Intel E5-2650) }\end{array}$} & 256 & $\approx 2^{14}$ & 192 bits & $42.7 \mathrm{k} / \mathrm{s}$ & 0.41 & $225 / \mathrm{J}$ & 0.034 \\
\hline & & & 256 bits & $27.8 \mathrm{k} / \mathrm{s}$ & 0.36 & $146 / \mathrm{J}$ & 0.030 \\
\hline & & & 384 bits & $13.9 \mathrm{k} / \mathrm{s}$ & 0.34 & $73 / \mathrm{J}$ & 0.028 \\
\hline & & & 512 bits & $8.65 \mathrm{k} / \mathrm{s}$ & 0.33 & $46 / \mathrm{J}$ & 0.028 \\
\hline & 1024 & $\approx 7 \cdot 2^{14}$ & 192 bits & $13.4 \mathrm{k} / \mathrm{s}$ & 0.49 & $71 / \mathrm{J}$ & 0.041 \\
\hline & & & 256 bits & $8.63 \mathrm{k} / \mathrm{s}$ & 0.43 & $45 / \mathrm{J}$ & 0.036 \\
\hline & & & 384 bits & $4.14 \mathrm{k} / \mathrm{s}$ & 0.39 & $22 / / \mathrm{J}$ & 0.034 \\
\hline & & & 512 bits & $2.58 \mathrm{k} / \mathrm{s}$ & 0.40 & $14 / \mathrm{J}$ & 0.034 \\
\hline & 8192 & $\approx 80 \cdot 2^{14}$ & 192 bits & $1.56 \mathrm{k} / \mathrm{s}$ & 0.45 & $8.2 / \mathrm{J}$ & 0.038 \\
\hline & & & 256 bits & $993 / \mathrm{s}$ & 0.40 & $5.2 / \mathrm{J}$ & 0.034 \\
\hline & & & 384 bits & $464 / \mathrm{s}$ & 0.38 & $2.5 / \mathrm{J}$ & 0.033 \\
\hline & & & 512 bits & $288 / \mathrm{s}$ & 0.38 & $1.5 / \mathrm{J}$ & 0.031 \\
\hline & 32768 & $\approx 360 \cdot 2^{14}$ & 192 bits & $372 / \mathrm{s}$ & 0.47 & $2.0 / \mathrm{J}$ & 0.040 \\
\hline & & & 256 bits & $240 / \mathrm{s}$ & 0.42 & $1.3 / \mathrm{J}$ & 0.036 \\
\hline
\end{tabular}

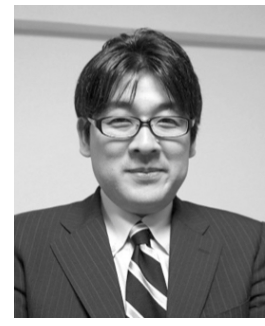

Atsuo Inomata received M.E. degree in information science from 1997 and 1999 and a Ph.D. in information science from Japan Advanced Institute of Science and Technology in 2002. Currently, he is a professor at Tokyo Denki University. His research focuses on cryptography, information security. $\mathrm{He}$ is a member of IEICE, IPSJ, and JSISE

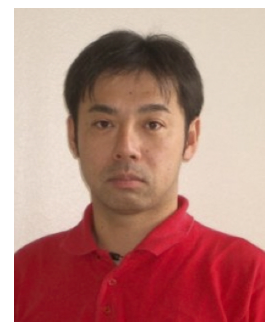

Kazutoshi Fujikawa received M.E. and Ph.D. degrees in information and computer sciences from Osaka University in 1990 and 1993, respectively. Currently, he is a professor of Information Initiative Center, Nara Institute of Science and Technology. His research focuses on multimedia communication systems, digital libraries, ubiquitous computing, and mobile networks. $\mathrm{He}$ is a member of ACM, IEEE, and IPSJ. 